Available online @ https://jiem.jnnce.ac.in https:www.doi.org/10.37314/JJEM.2021.050202 Indexed in International Scientific Indexing (ISI) Impact factor: 1.395 for 2021-22 Published on: 31 January 2022

\title{
IOT Based Smart Poultry Farming
}

\author{
Ananya M D ${ }^{1}, \quad$ Sudeep Manohar ${ }^{2 *}$ \\ 1,2Department of Information Science and Engineering \\ Jawaharlal Nehru National College of Engineering, Shivamogga, Karnataka, India \\ 1'mdananya82@gmail.com, $\quad{ }^{2}$ sudeepmanohar@jnnce.ac.in
}

\begin{abstract}
Nowadays, automation plays a very important role in everyday life. IoT based poultry farming is useful in situations where remote monitoring and maintenance is needed and this in turn modifies the conventional farm into a modern farm with various automated features. Various factors that affect the health of chicken are monitored at regularly interval to improve health and growth of chicken. This paper provides an insight on how to setup an IoT based Smart Poultry Farming System using IoT components. The system uses Arduino Nano for interfacing with different sensors to sense the values of specific parameters and ESP8266 Wi-Fi Module to upload the data to the cloud. Varous environmental factors are sensed in the poultry management system, which includes temperature, ammonia content in the air and light intensity. The system not just monitors these parameters, but also regulates them effectively with the help of various automated techniques. The proposed work is useful to farmers who follow conventional farming methods as they could easily access and control the poultry farm remotely using their mobile phones thereby reducing the manual monitoring and increasing the yield of the poultry farm.
\end{abstract}

Keywords: Arduino Nano, ESP8266, IFTTT, Sensors, ThingSpeak

\section{Introduction}

India is an agriculture rich country and people here grow various crops and raise animals which are of much use to the people. Animal husbandry or Livestock farming is a part of agriculture where animals are raised. Poultry farming is one of the leading Livestock farming which has given financial support for many farmers in the country. Chicken is one of the most liked food in the world. It is rich in proteins and nutrients. The low levels of fat and cholesterol makes it a healthy food for most of the health-conscious people around the globe. Even though India produces chicken in large numbers, the conventional methods followed in poultry farming has decreased the yield, thereby resulting in poor financial gains. In the recent years poultry farming has seen a considerable growth by following latest farming methods. This has led to increase in domestic and international export. As people become literate, they usually divert from agriculture practices and hence there creates lack of laborers to monitor and maintain the poultry farms. Hence there is a need to automate the poultry farm by incorporating new technologies that are trending in the market, which can increase the yield of the farm. IoT Based Smart Poultry Farming is one of the solutions that can be adopted in poultry farming. Using IoT in farming solves the problem of unavailability of laborers and also increases the efficiency of the farm, thereby increasing the poultry yield. IoT devices are economically feasible and easily available that can be adopted by all farmers.

The following are the objectives of the proposed work and the necessary implementation is carried out to accomplish the set target. First, the traditional way of 
poultry farming is replaced with the smart and intelligent farming using IoT based technologies as the former method incurs more work force and less yield. As the technology is available at a very low budget it is economically feasible to deploy in almost all poultry farms without financial overhead.

Second, it helps the farmers in real time controlling and monitoring of environmental aware context parameters such as temperature, air quality and light intensity by using corresponding regulating devices like heaters, exhaust fans, and lights. Third, this smart system is used to remotely control the farm from any location which reduces the amount of time and manual work consumed. As the system is connected to internet, it becomes easy for an individual to keep track of the poultry environment conditions wherever he is. Finally, the system will improve productivity and quality of chickens in poultry farming by providing healthy and necessary environment for its growth.

Internet of Things (IoT) [1] connects many devices together with the internet, that sense and control various components is a network. The data sent is processed [2] and stored in the cloud or is used to actuate other IoT devices. IoT devices are portable and easy to access which comes with less cost and consumes less power. IoT provides platform for Machine to Human communication $(\mathrm{M} 2 \mathrm{H})$ and Machine to Machine (M2M) communication.

The proposed work focuses on building a system setup using latest technologies [3] like IoT, low cost hardware and free software which include monitoring and controlling of farms [4]. The system makes use of specific sensors to sense noticeable changes in its surroundings and notifies the concerned person on his cell phone using an app. A threshold is set for all the parameters and when the sensed data crosses this threshold, corresponding devices can be actuated remotely to bring the value below threshold. Thus we can save time and increase the hygiene in the locality [5].

\section{System Architecture}

Architecture for the IoT Based Smart Poultry Farming is as shown in Figure 1. Arduino Nano micro-controller is used which is connected to ESP8266 and relay. The sensor readings received by Arduino is passed to ESP8266 which acts as Wi-Fi module. Arduino accepts data from various sensors that are connected. The algorithm compares the read values with the threshold and sends actuating signals to the relays connected to the Arduino. The relays switches $\mathrm{ON}$ or $\mathrm{OFF}$ and the connected devices operate accordingly. A $5 \mathrm{~V}$ adapter is used in order to power up the Arduino and a $12 \mathrm{~V}$ battery is used in order to power the devices like bulb and exhaust fan. The architecture block diagram is shown in Figure 2.

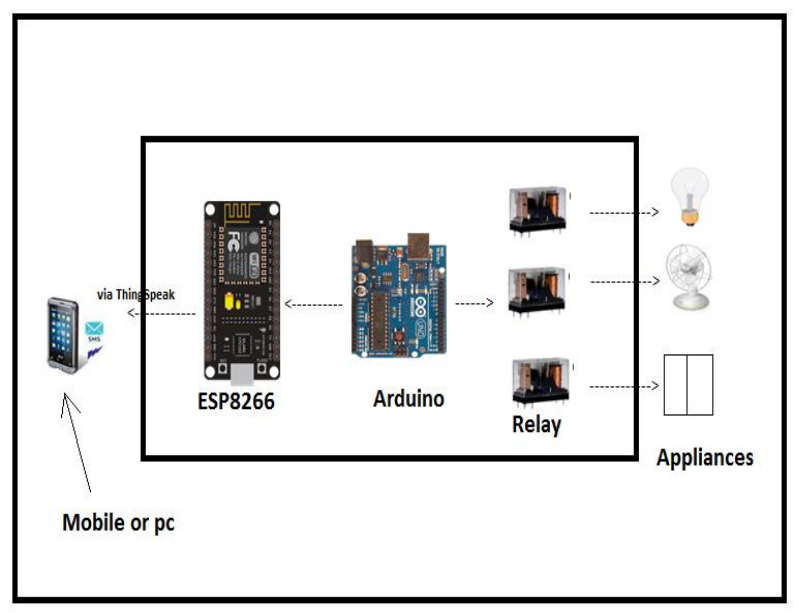

Figure 1: System Architecture of proposed System

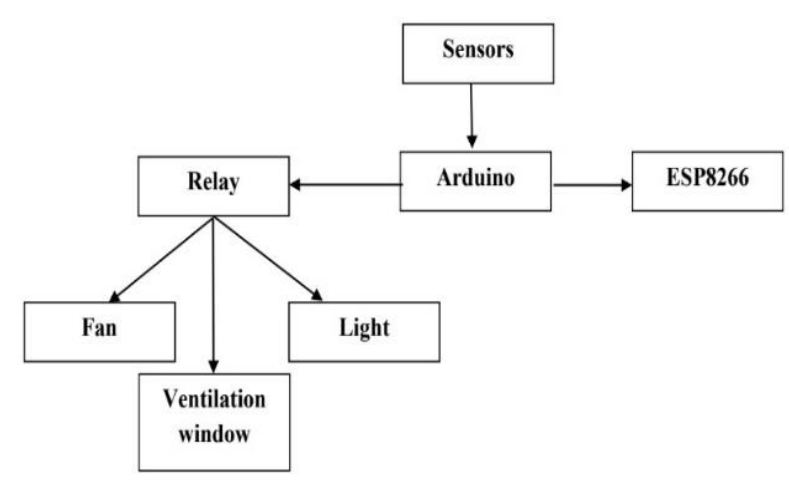

Figure 2: System architecture block diagram 


\subsection{Arduino Nano}

Arduino Nano is a programmable micro controller board which has onboard ATmega329P chip used for computing purpose. The 14 digital pins and 6 analog pins are used for data input and output. It is equipped with $16 \mathrm{MHz}$ quartz crystal which generates the needed frequency. The pins of the Arduino Nano can be used as input or output using the functions, pinMode(). The Arduino Nano can communicate with a computer or any other IoT device. Arduino IDE is used to write program for Arduino boards. The board is powered up using USB cable or external batteries or power supply.

\subsection{ESP8266}

NodeMCU is a microcontroller which runs on ESP8266 SoC [6]. This board is equipped with analog and digital pins which are used for reading and writing data. The board also can connect to any Wi-Fi access point using the onboard Wi-Fi module. Application which needs wireless connectivity can make use of this board.

\subsection{Channel 5V Relay}

A relay is electronically operated to perform the $\mathrm{ON}$ and $\mathrm{OFF}$ operations. An electromagnet is used to operate a relay. These are usually operated at low signals using the output of Arduino or NodeMCU. Various home electrical equipment like bulb, fan, refrigerator etc. can be controlled remotely by using relays. Arduino program sends signals which turns ON/OFF the target appliance.

\subsection{DHT11 Temperature Sensor}

DHT11 is used to sense the room temperature and humidity. A capacity humidity sensor and a thermistor is used to measure the values in the surroundings [7]. It outputs digital output of temperature in centigrade and humidity in percentage. For its proper operation, no instruction should be sent to it within one second after powering ON. And a capacitor of calculated value needs to be connected between VCC and GND.

\subsection{MQ-135 Gas Sensor}

MQ-135 sensor is used to sense the concentration of various gases in the environment. This sensor contains 3 pins and gives analog output. This can sense ammonia, ammonium oxide, alcohol, benzene, carbon dioxide and smoke. Since this sensor is highly sensitive to ammonia, it is used in the proposed work. This sensor is used in various applications where quality of air has to be measured.

\subsection{LDR Photo resistor}

A Light Dependent Resistor (LDR) is controlled by the light that is incident on it. The resistance of LDR decrease as the light increases. Various applications where light sensing is required makes use of this resistor.

\subsection{ThingSpeak}

ThingSpeak is an open source software which is used to read and write data from IoT devices using HTTP. Data logging for various applications can be done using ThinkSpeak. The data that is stored in the web can later be read and processed by another IoT device to take actions. ThinkSpeak provides facility to plot different types of graphs from the data that is stored.

\subsection{IFTTT}

If This, Then That (IFTTT) is used in scenarios where conditional statements are needed in IoT application. It is a web base free service which can do small tasks online for the applications. These conditional tasks are called applets. IFTTT employs the following concepts:

- "this" part of the applet "triggers" the action

- "that" part of the applet specifies the action 
A sensor can be considered as a trigger which reads the data. If the sensed values exceed the threshold value, then an action triggers which performs a specific task. The conditional checking is done online based on the data that is fed into the cloud using ThingSpeak.

\section{System Implementation}

The IoT based Smart Poultry Farming System is implemented using IoT hardware devices and software. Various sensors are used to sense the environmental parameters such as light intensity, ammonia concentration and temperature. The sensed data is uploaded to cloud using NodeMCU. IFTTT service is used to monitor the data that is sensed. When a data value reaches a threshold, certain actions are triggered that actuates particular devices using relay module.

The NodeMCU module communicates with the concerned person who is in-charge of the poultry farm. IFTTT service triggers suitable actions to send messages to the person and to other devices. The NodeMCU sends data that is read from the sensors to the cloud using ThingSpeak. Data analysis is done by plotting graphs. The graphs plotted can be viewed on any computer or handheld devices using internet. The analysis can be made use of the poultry farm in-charge to take actions which could enhance the productivity of the farm.

The implementation is done on a smaller model of the poultry farm whose environment depicts the real environment. Sensors, which can cover a smaller geographical area, are considered. The temperature is varied by applying heat near the DHT 11 sensor and when the sensor detects a raise in temperature, the algorithm actuates the relay to turn $\mathrm{ON}$ the exhaust fan to lower the temperature inside the poultry farm. The light intensity on the LDR is reduced by covering it by some object. The LDR senses the decrease in intensity of light and the algorithm turns $\mathrm{ON}$ the bulb. And as the intensity of light is increased, the bulb is turned OFF automatically. To see the effect of gas, ammonia is used near the gas sensor, which detects the percentage of ammonia in the air and sends the data to the microcontroller. The algorithm checks the data, compares and if it crosses the threshold, the fan is switched $\mathrm{ON}$ to reduce the concentration of ammonia in the poultry farm. The whole module is connected to ThinkSpeak which can read the sensor values directly to web and can display the status of the read values in the poultry farm. ThinkSpeak can be accessed from anywhere through the web which enable the person in-charge of the poultry farm to monitor the various parameters of the environment.

\subsection{Algorithm}

Step 1. Start the process

Step 2. Acquire the data from the sensor

Step 3. Sensor data is input to Arduino Nano

Step 4.1: When temperature goes beyond threshold value then cooling fan will be turned ON automatically.

Step 4.2: When percentage of ammonia in gas goes beyond threshold value then ventilation window will be opened automatically.

Step 4.3: When light intensity goes below threshold value then light will be switch $\mathrm{ON}$ automatically.

Step 5: Connect ESP8266 Wi-Fi module to Arduino Nano. This helps to upload data to the web page.

Step 6: The person in-charge of the poultry farm can get the internal environmental conditions through mobile/PC using Internet.

\section{Results and Discussion}

The system has been implemented and tested for changing environmental conditions. System setup includes sensors, microcontroller, Wi-Fi module and actuators. Figure 3 shows the connection between the various devices. The temperature, light and gas values are varied by making suitable environmental changes. The read values from the sensors are processed and results obtained are observed against expected outcome. 


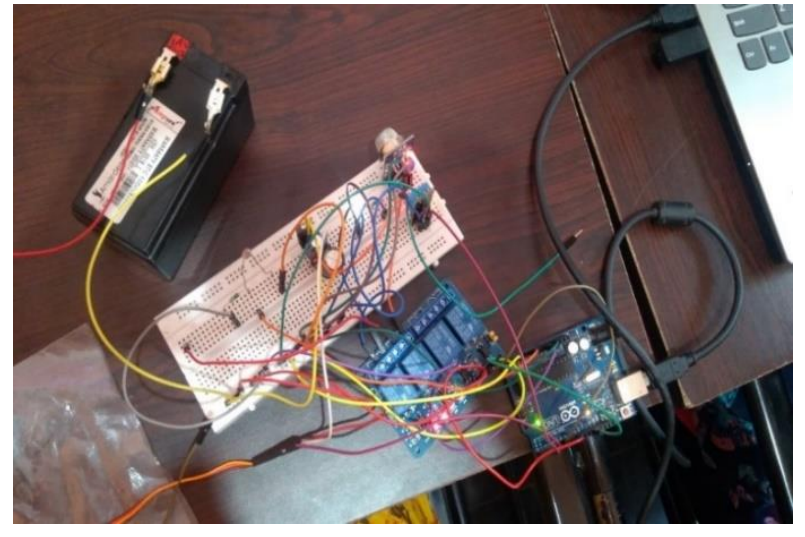

Figure 3: Connection between aggregator, coordinator node and actuator nodes

Figure 4 shows the graphical output of the values read by the sensors through ThinkSpeak application. The graph is helpful in providing timely data to the person who is in-charge of the poultry farm. This graph may also be used for analysis purpose for future scope.

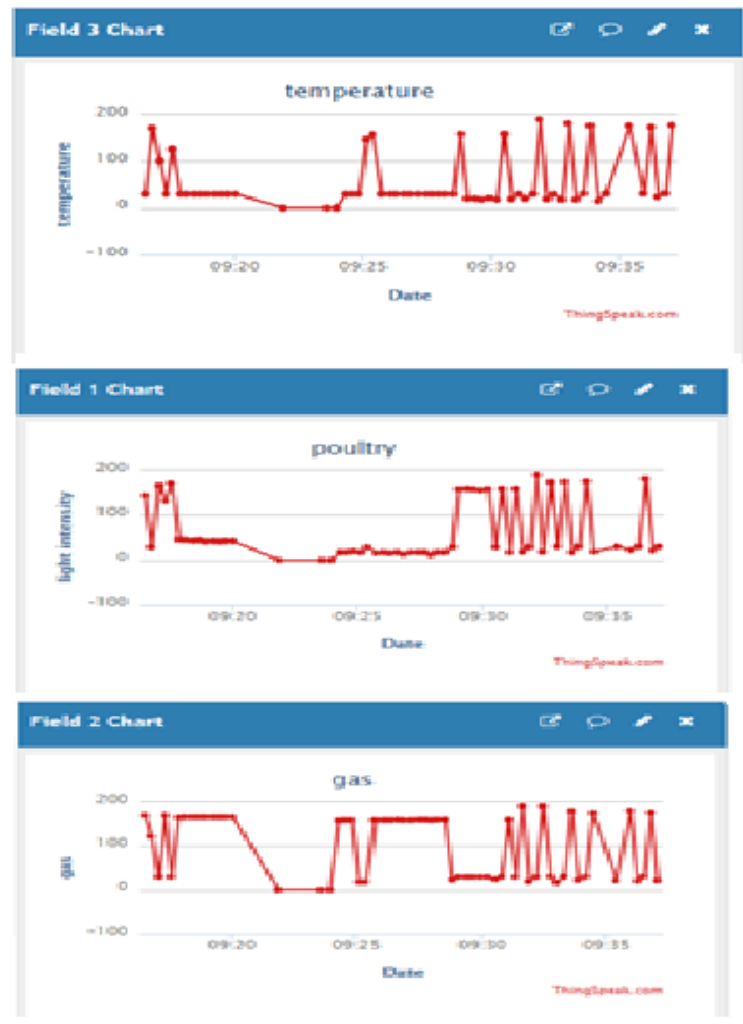

Figure 4: shows the graphical analysis of all the sensors over a period of time implemented using Thingspeak IoT.
Whenever a physical parameter crosses a predefined value, a notification is sent to the person as shown in Figure 5. The algorithm checks the sensed data and if it is out of the threshold level, the poultry farm personnel is informed about the change in the environmental conditions through mail and SMS. This alert system enables the person to take action against the change and can save the chicken from any disaster caused due to temperature or light or gas level.
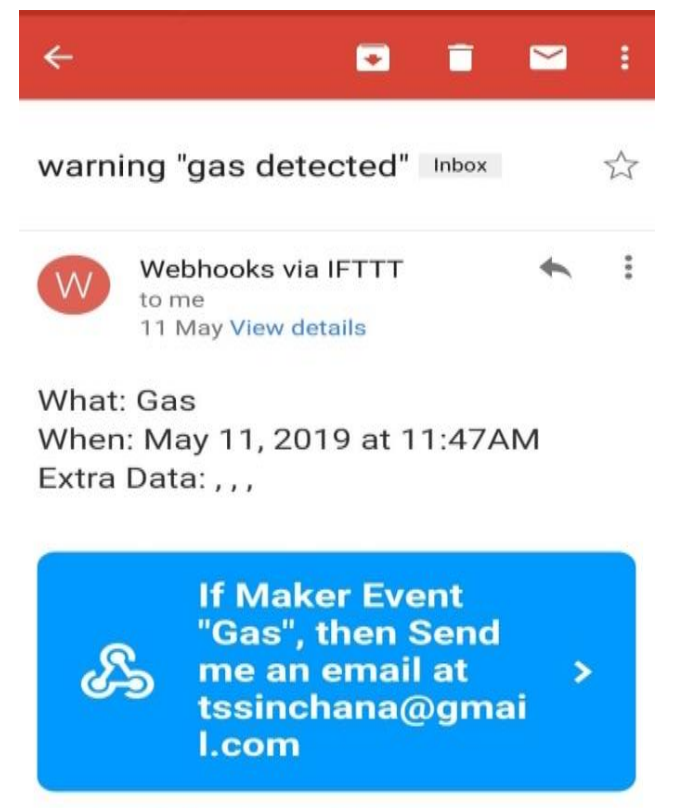

Unsubscribe from these notifications or sign in to manage your Email Applets.

$$
112 \cos =
$$

Figure 5: Email notification sent to the owner when gas is detected

\section{Conclusion}

The proposed work demonstrates an IoT system setup that can be used in poultry farms. It comprises of sensors, Arduino Nano microcontroller, ESP8266 Wi-Fi module and relays. The system is easy to adopt and requires less modification and investment. The parameter values can be monitored remotely using a smart phone. The level of temperature, ammonia gas and light can be controlled automatically using actuators. Since close monitoring is done in this proposed system, health and growth of chickens are improved. 
Most of the labor work is reduced thereby increasing productivity of the poultry farm. The idea can be enhanced to design water level control and food control mechanism with the help of sensors. The proposed work can be extended to detect dead and diseased chicken by capturing their movements by using cameras and thermal sensors in the poultry farm.

\section{References}

1. Srinivasa $\mathrm{K} G$, Internet of Things, CENGAGE Learning Indian, 2007.

2. Eric Hitimana, Gaurav Bajpai, Richard Musabe, Louis Sibomana, Remote monitoring and control of poultry farm using IoT techniques, International Journal of Latest Technology in Engineering, Management \& Applied Science (IJLTEMAS), Vol.7, Issue 5, May 2018, pp.87-90.

3. Ahmad Ammar Nor Azlin, Hasmah Mansor, Ahmad Zawawi Hashim, Teddy Surya Gunawan, Development of Modular Smart Poultry Farm System, Proceedings of the IEEE 4th International Conference on Smart Instrumentation, Measurement and Application (ICSIMA), 28-30 Nov. 2017, Putrajaya, Malaysia, DOI: 10.1109/ICSIMA.2017.8312019.
4. Carlos González, Ricardo Pardo, José Fariña, María D. Valdés, Juan J. Rodríguez Andina, Manuel Portela, Real-Time Monitoring of Poultry Activity in Breeding Farms, Proceedings of the 43rd Annual Conference of the IEEE Industrial Electronics Society, IECON 2017, 29 Oct.-1 Nov. 2017, Beijing, China, DOI: 10.1109/IECON.2017.8216605

5. Arun Gnana Raj, J. Gnana Jayanthi, IoT Based Real-time poultry monitoring and Health Status Identification, Proceedings of the 11th International Symposium on Mechatronics and its Applications (ISMA), 4-6 March 2018, Sharjah, United Arab Emirates, DOI: 10.1109/ISMA.2018.8330139

6. David Hanes, Gonzalo Salgueiro, Patrick Grossetete, IoT Fundamentals: Networking Technologies, Protocols and Use Cases for the Internet of Things. Cisco Press 800 East 96th Street Indianapolis, Indiana 46240 USA

7. Ricardo Pardo; Carlos González; José Fariña; María D. Valdés; Juan J. Rodríguez Andina; Manuel Portela, Measurement of Air Flow in New born Poultry Transportation system, Proceedings of the 43rd Annual Conference of the IEEE Industrial Electronics Society, 29 Oct.-1 Nov. 2017 Beijing, China , DOI: 10.1109/IECON.2017.8216604 